\title{
Simulations of Needle Insertion by Using a Eulerian Hydrocode FEM and the Experimental Validations
}

\author{
Hiroyuki Kataoka, Shigeho Noda, Hideo Yokota, Shu Takagi, Ryutaro Himeno, \\ and Shigenobu Okazawa \\ Research Program for Computational Science, RIKEN, \\ 2-1 Hirosawa, Wako-shi, Saitama, 351-0198 Japan \\ Graduate School of Engineering, Hiroshima University, Japan \\ \{h.kataoka, shigeho, hyokota, takagish, himeno\} @riken.jp, \\ okazawa@hiroshima-u.ac.jp
}

\begin{abstract}
In this paper, simulations for needle insertion were performed by using a novel Eulerian hydrocode FEM, which was adaptive for large deformation and tissue fracture. We also performed experiments for the same needle insertion with silicon rubbers and needles, which had conical tips of different angles in order to investigate the accuracy of the simulations. The resistance forces in the simulations accurately followed those in the experiments until the conical portion of the needle was inside the rubbers, and the validation of the Eulerian hydrocode was revealed. However, the present simulation showed that after the conical portion was inside the tissue, the simulated resistance forces became lower than the experimental ones. The proportional increase of the friction forces and the roughly flatness of the tip force along the time were simulated. It was predicted that the tightening force along the needle side was underestimated.
\end{abstract}

Keywords: needle insertion, FEM, Eulerian hydrocode, large deformation, friction.

\section{Introduction}

Needle insertion is one of the procedures with least invasive in surgical treatments. However, it is a common problem for surgeons to precisely control the needle path to a target deep inside tissues even with the help of medical images, because soft tissues keep deforming with the inserting of a needle, and the thinner needles can also deflect. Therefore, preoperative simulations of tissue deformation and needle deflection would be helpful to optimize the needle path, and for training of surgical procedures [1-2].

In the present simulations with Finite Element Method (FEM), Lagrangean mesh is defined in an object, and warps following the deformation of the object (Fig.1a). In the case of the needle insertion, large deformation of soft tissues possibly occurs by the needle, and as the results, the simulation may not be able to proceed because of unexpected numerical collapse of the elements. In addition, when the edges of the 
needle tip tear the tissues, mesh modification is required to separate the corresponding tissue elements into two different elements along the edges.

A novel FEM named Eulerian hydrocode (EH) [3-4] has been developed against large deformation problems such as plastic forming. In this method, Eulerian mesh is defined in the space, and never warps (Fig.1b). The object is represented as filling ratio at each element, and the deformation of the object is achieved by drift of the filling ratio to the neighbor elements as like fluid according to both Euler equation and constitutive equations for solid. Therefore, the deformation of the object follows solid mechanics. The tear of the object can also be performed following the fracture of an element, where the filling ratio is removed instead of the mesh modification.

In this study, we introduced the EH to the simulations of the needle insertion, and we assembled the contact and friction forces on the needle surface and the tissue fracture by at the needle edges. We also validated the accuracy of the simulations by comparing the resistance forces of the needles between in the simulations and phantom experiments with silicon rubbers.

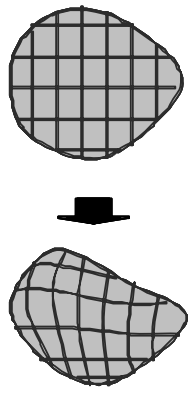

(a)

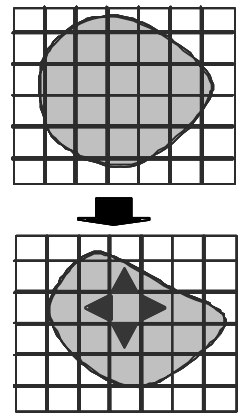

(b)

Fig. 1. (a) Lagrangean and (b) Eulerian mesh
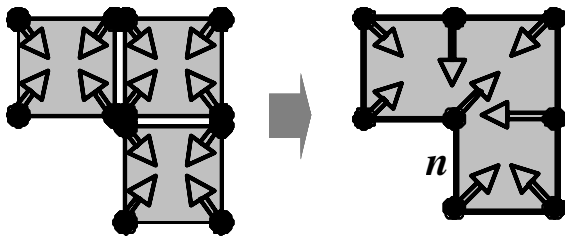

Fig. 2. Generation of the inward normal vectors $\boldsymbol{n}$ on the needle surface from diagonal vectors for each element

\section{Materials and Methods}

\subsection{Algorithm of Eulerian Hydrocode}

The Eulerian hydrocode (EH in abbreviation) is based on the general relation between the material and spatial time derivatives in a potential field $\phi$, which is represented as

$$
\frac{D \phi}{D t}=\frac{\partial \phi}{\partial t}+v \cdot(\nabla \phi)
$$

where $v$ is material velocity, $D \phi / D t$ and $\partial \phi / \partial t$ are material and spatial time derivatives of $\phi$, respectively. The material time derivative is formulated in the standard Lagrangean solid mechanics as the mass, momentum, and energy equations, respectively. By substituting each material time derivative into Eq.1, Eq.1 can be rewritten in a general form as, 


$$
\frac{\partial \phi}{\partial t}+\nabla \cdot \boldsymbol{\Phi}=S
$$

where $\boldsymbol{\Phi}$ is the flux function, and $S$ is the source term.

In the EH, Eq.2 is separated into Lagrangian step (Eq.3) and Eulerian step (Eq.4) using operator splitting method.

$$
\begin{gathered}
\frac{\partial \phi}{\partial t}=S \\
\frac{\partial \phi}{\partial t}+\nabla \cdot \boldsymbol{\Phi}=0
\end{gathered}
$$

The Lagrangian step can be identical to the standard Lagrangian governing formulations by replacing the spatial time derivative to the material time derivative. In our EH, Eq.3 was formulated with a constitutive equation for elasto-plastic materials (Eq.5) using updated Lagrangian formulation and dynamic explicit method,

$$
\stackrel{\circ}{T}=C^{e p}: D
$$

where $\stackrel{\circ}{T}$ was Jaumann stress rate tensor, $D$ was deformation rate tensor, $C^{\text {ep }}$ was a material coefficient tensor. Considering that the plasticity of most soft tissues was unknown, we ignored the plasticity, and applied the standard elastic coefficient tensor to $C^{\mathrm{ep}}$, which consisted of Young's Modulus and Poisson's ratio. The Eulerian step moves the deformed mesh at the Lagrangian step to the original fixed mesh by transporting the mass, momentum, and stress to the neighbor elements. In our Eulerian step, MUSCL scheme [5] was used for the advection scheme of Euler equation. For more details of the EH, please refer Refs [3-4].

\subsection{Configurations of Eulerian Hydrocode for Needle Insertion}

For the present work of out EH, the single object could be handled. We fixed the needle in the space, and represented the needle surface as wall boundary condition. Then, the tissue was moved toward the needle instead of moving the needle.

Firstly, we calculated contact force between the needle and tissue to estimate the friction force and total resistance force acting on the needle surface. In this study, the needle was defined as a group of voxel. Therefore, in order to estimate the inward normal vectors $\boldsymbol{n}$ to the needle on the needle surface, we calculated a unit diagonal vector at each node for each voxel, and summed all the vectors for each node as depicted in Fig.2. The inward normal components of the nodal velocities on the needle surface were removed as the wall boundary condition using $\boldsymbol{n}$. Since this change of the nodal velocities was caused by the contact force on the needle surface, we estimated the nodal contact force $\boldsymbol{F}_{n}$ from the reduction of momentum of the tissues as Eq.6,

$$
\boldsymbol{F}_{n}=\frac{m v \cdot \boldsymbol{n}}{\Delta t} \cdot \boldsymbol{n}
$$


where $m$ was the concentrated nodal mass, $\boldsymbol{v}$ was nodal velocity, $\boldsymbol{n}$ was the normalized inward normal vector, and $\Delta t$ was time step.

Secondly, we estimated the nodal friction force $\boldsymbol{F}_{\mathrm{t}}$ on the needle surface. The unit vector in the direction of the parallel component of the nodal velocity to the needle surface was defined as $\boldsymbol{t}$. Then, we assumed Coulomb friction and the state transition between fixing and slipping states of the needle-tissue interaction. At the fixing state, there was no relative speed between the needle and tissue. Therefore, $\boldsymbol{F}_{\mathrm{t}}$ was estimated as Eq.7 to cancel the nodal velocity in the $\boldsymbol{t}$ direction, as well as Eq.6.

$$
\boldsymbol{F}_{t}=-\frac{m \boldsymbol{v} \cdot \boldsymbol{t}}{\Delta t} \cdot \boldsymbol{t} \quad\left(\left|\boldsymbol{F}_{t}\right|<\mu \mid \boldsymbol{F}_{n}\right)
$$

When the shear increases, and the magnitude of $\boldsymbol{F}_{\mathrm{t}}$ exceeds the upper limitation proportional to the magnitude of $\boldsymbol{F}_{n}$, the fixing state switches to the slipping state. $\boldsymbol{F}_{\mathrm{t}}$ at the slipping state was estimated as Eq.8 with the dynamic friction coefficient $\mu$, and worked as an external force to reduce the nodal velocity in the $t$ direction.

$$
\boldsymbol{F}_{t}=-\mu\left|\boldsymbol{F}_{n}\right| \cdot \boldsymbol{t} \quad\left(\left|\boldsymbol{F}_{t}\right|=\mu\left|\boldsymbol{F}_{n}\right|\right)
$$

The total resistance force acting on the needle $\boldsymbol{F}_{\text {total }}$ was estimated by summing up all $\boldsymbol{F}_{n}$ and $\boldsymbol{F}_{\mathrm{t}}$, and recorded to files during the simulation process with $\mathrm{EH}$.

We also simulated the tissue fracture at the needle edge. For doing so, the voxel contacting to the cutting edges of the needle were preliminarily defined, and the von Mises stress at these voxel were monitored. Then, when the von Mises stress exceeded a pre-defined threshold in a voxel, we regarded that the voxel fractured, and both the filling ratio and Cauchy stress of the voxel were set to be 0 .

\subsection{Experimental Setup}

In order to validate the results of the simulations, we performed experiments of needle insertion with silicon rubbers and large scaled needle. Cylindrical silicon rubbers (KE-14, Shin-Etsu Chemical Co. Ltd., Tokyo, Japan) of the diameter about $80 \mathrm{~mm}$ were prepared. Young's modulus of the rubbers was estimated as $0.545 \mathrm{MPa}$ from the preliminary uniaxial loading tests. Needles of the diameter $7 \mathrm{~mm}$ were prepared from brass shafts, whose tips were conical with three different angles of 30, 60 and 90 degree, as shown in Fig.3. The tips were lubricated with oil. Each needle was attached to the loading machine (Autograph AGS-H, Shimadzu Co., Kyoto, Japan) (Fig.4), and moved back and forth five times into the silicon rubbers at a constant speed of $5 \mathrm{~mm} / \mathrm{sec}$ until the insertion distance reached to $10 \mathrm{~mm}$ or the resistance force reached to $45 \mathrm{~N}$, which was the limitation of the load cell. The resistance force in the axial direction of the needle was measured at $20 \mathrm{~Hz}$ sampling rate.

\subsection{Simulation Setup}

The experiments were simulated by using EH. Considering that the diameter of the dent on the rubber surface by the needle was observed within twice of the needle diameter in the above experiments, a rectangular solid of the size $20 \times 20 \times 13.5 \mathrm{~mm}$ for the silicon rubbers was located at the bottom of the calculation space of the size 
$22 \times 22 \times 25 \mathrm{~mm}$. A rigid needle of the diameter $7 \mathrm{~mm}$ with the conical tips of angles of 30, 60, and 90 degrees was also located above the solid, as shown in Fig.5. The voxel size was $0.5 \times 0.5 \times 0.5 \mathrm{~mm}$. The solid was moved upward at the speed of $5 \mathrm{~mm} / \mathrm{sec}$ by giving the same velocity to the nodes at the tissue bottom.

The rubber is considered nearly incompressible, and Poison's ratio between 0.48 0.5 is commonly used. However, since our EH required unexpectedly smaller time step for more close value to 0.5 , we set Poisson's ratio as 0.47 after some trials. We set the friction coefficient as 0.3 according to the results for steel-rubber friction with oil lubrication at 25 degree temperature and $20 \mathrm{~N}$ load in the Ref [6]. Total simulation time was set as $2.0 \mathrm{sec}$ for $10 \mathrm{~mm}$ insertion considering the experiments.

The time step $\Delta t$ had to be determined enough small to satisfy Courant-FriedrichsLewy (CFL) condition. In the case of solid, this condition depended on the propagation rate of the stress in the solid, and the stiffer and smaller density material generally required the smaller $\Delta t$. In a trial simulation, our $\mathrm{EH}$ required $\Delta t=2 \times 10^{-6} \mathrm{sec}$ for true Young's Modulus $(E=0.545 \mathrm{MPa})$ and density $\left(\rho=1.0 \mathrm{~g} / \mathrm{cm}^{3}\right)$, and the total calculation time was estimated about 90days. Therefore, in this study, we considered to increase $\Delta t$ by increasing $\rho$. The increase of $\rho$ had an effect to reduce acceleration according to the equation of motion. Then, the transient response of the solid for higher frequency vibration would be reduced, and the simulation became close to quasi-static.

Considering the total calculation time within a day, we finally scaled $\rho$ as $1.0 \times 10^{4} \mathrm{~g} / \mathrm{cm}^{3}$, which was $10^{4}$ times as large as the true value for the silicon rubbers, and we applied $\Delta t=2.0 \times 10^{-4} \mathrm{sec}$ to the simulation. We also investigated the influence of the density scaling and the approximation to the quasi-static analysis. We compared the resistance forces for the tip angle of 90degree between with the different $\rho$ scaling of $10^{2}-10^{6}$. The time steps and total calculation times were represented in Table 1.

In terms of the threshold of von Mises stress for the tissue fracture, we tested $0 \mathrm{~Pa}$ for the most acute case, and infinity for the case without fracture. Only one voxel at the apex of the conical tip was defined for the tissue fracture operation.

All the calculations were performed on a PC (CPU: Intel dual core Xeon $2.6 \mathrm{GHz}$, Memory: 4Gbyte) with single process.

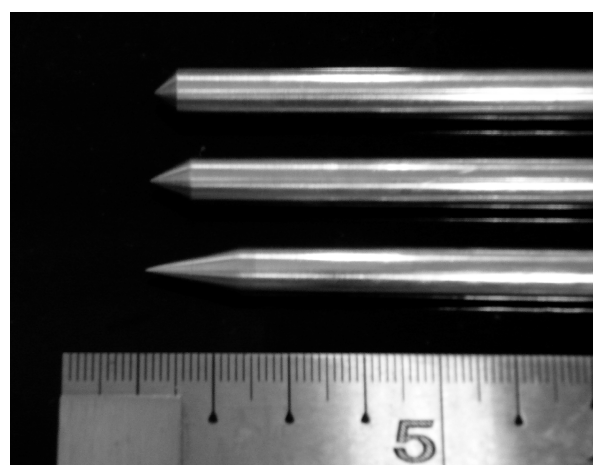

Fig. 3. Needles with the conical tips, and the tip angle are 30,60, and 90 degree, respectively

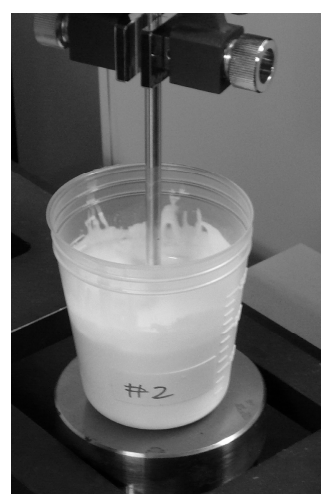

Fig. 4. Apparatus of the phantom experiments for the needle insertion 
Table 1. The series of density, time step, total steps, and calculation time used for the present simulations

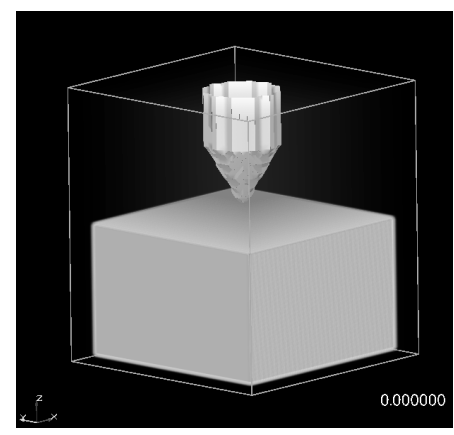

\begin{tabular}{c|c|c|c|c|c} 
Density $\left(\mathrm{g} / \mathrm{cm}^{3}\right)$ & $1.0 \times 10^{2}$ & $1.0 \times 10^{3}$ & $1.0 \times 10^{4}$ & $1.0 \times 10^{5}$ & $1.0 \times 10^{6}$ \\
\hline Time step & $2 \times 10^{-5}$ & $5 \times 10^{-5}$ & $2 \times 10^{-4}$ & $4 \times 10^{-4}$ & $8 \times 10^{-4}$ \\
\hline Total steps & 120000 & 48000 & 12000 & 6000 & 3000 \\
\hline Calculation time & $262 \mathrm{~h}$ & $104.8 \mathrm{~h}$ & $26.2 \mathrm{~h}$ & $13.1 \mathrm{~h}$ & $6.55 \mathrm{~h}$
\end{tabular}

Fig. 5. Initial geometrical condition of the simulations

\section{Results}

The distributions of the filling ratio and von Mises stress in a vertical section under the needle insertion for the tip angle 60degree at 2.1sec are shown in Fig.6 as a sample of the simulation results. It was a feature of $\mathrm{EH}$ that the filling ratio at the solid boundary was blurred.

The axial components of the experimental and simulated resistance forces to the needle with and without friction are shown in Fig.7-9. Since the experimental forces were quite different between at the first and the rest in 5 insertions, we plotted the last 4 forces. For the tip angles of 60 and 90degree, the conical portion of the needle become inside of the silicon rubbers at about 1.22 and $0.70 \mathrm{sec}$, respectively. Before the conical portion was inside the solid, the simulated resistance forces accurately followed the experimental forces. On the contrary, after the conical portion was inside the solid, the simulated forces were lower than the experimental ones. It was observed that the silicon rubbers tore for the tip angles of 30 and 60 degree in the experiments.

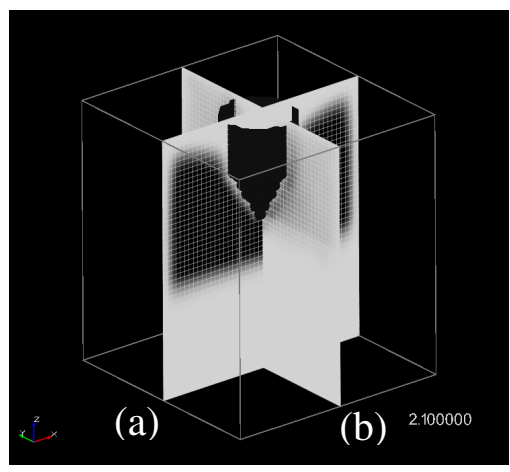

Fig. 6. The distribution of the (a) filling ration and (b) von Mises stress in the vertical sections for the tip angle of 60degree at $2.1 \mathrm{sec}$

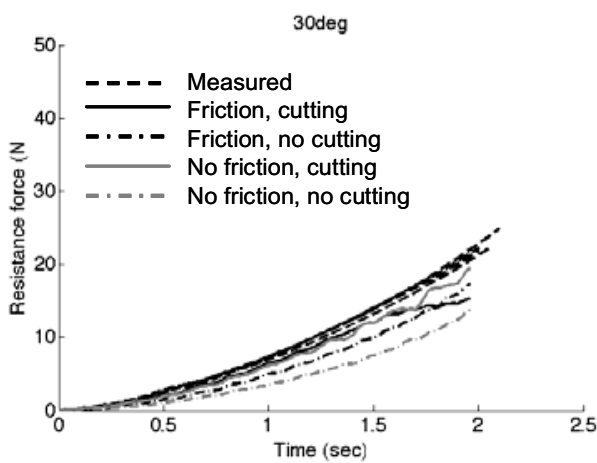

Fig. 7. The axial component of the experimental and simulated resistance forces of the needle for the tip angle of $30 \mathrm{degree}$ 


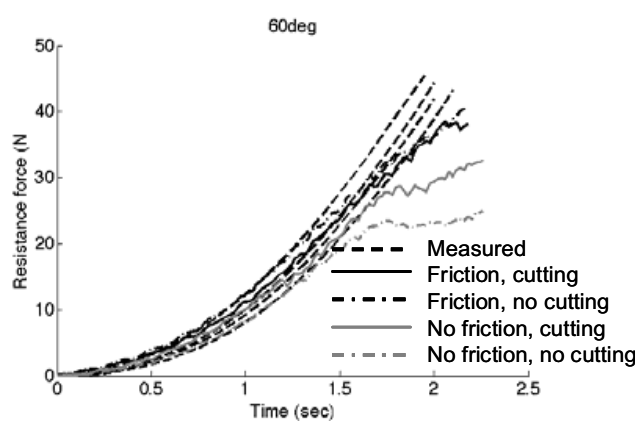

Fig. 8. The axial component of the experimental and simulated resistance forces of the needle for the tip angle of 60degree

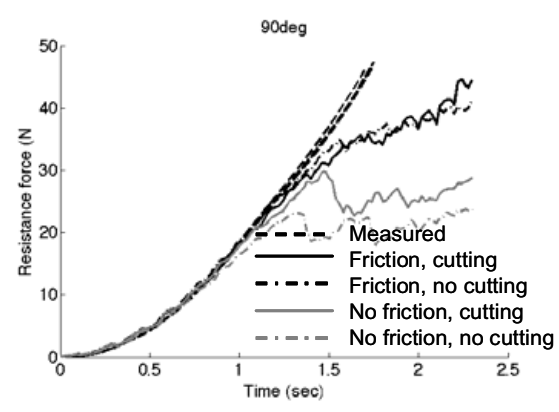

Fig. 9. The axial component of the experimental and simulated resistance forces of the needle for the tip angle of 90degree

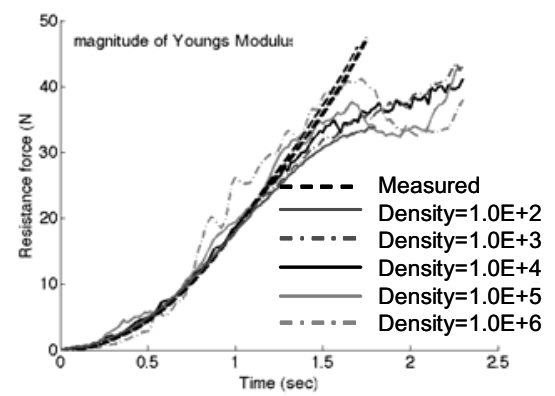

Fig. 10. The axial component of the experimental and simulated resistance forces of the needle for the tip angle of 90degree with different density increase ratios

The influence of the density scaling to the resistance forces is shown in Fig.10. The forces had no significant difference between the different scaling, though the smoothness of the curves increased for the densities close to the real value.

The calculation time for each time step for 96800 voxel was about $7.86 \mathrm{sec}$, which was proportional only to the number of voxel. The total calculation time for the density $1.0 \times 10^{4} \mathrm{~g} / \mathrm{cm}^{3}$ with total steps of 12000 was about 26.2 hours.

\section{Discussion}

The simulated resistance forces $\boldsymbol{F}_{\text {total }}$ for the different density scaling and the experimental $\boldsymbol{F}_{\text {total }}$ in Fig.10 had no significant difference until the conical portion of the needle was inside the solid. This could prove that the needle insertion could be simulated as approximately quasi-static, and it was revealed that the use of the density scaling was effective to increase the time step $\Delta t$. However, since the larger noise occurred for the larger density, smaller $\Delta t$ was still recommended, and the trade-off between the total calculation time and the accuracy should be considered. 
Before the conical portion of the needle was inside the solid, the simulated $\boldsymbol{F}_{\text {total }}$ were better matched with the experimental $\boldsymbol{F}_{\text {total }}$, and it was considered that the simulation by the EH worked accurately. However, the accuracy slightly decreased for the smaller tip angle. It was supposed that the reason was the error of shape representation for the sharp tip using a group of cubic voxel. Though the increase of the geometric resolution could avoid this problem, and increase the accuracy of the simulations with less blur of the solid boundary, the higher resolution had a problem of the increase of the total calculation time.

After the conical portion was inside the solid, the simulated $\boldsymbol{F}_{\text {total }}$ was underestimated compared with the experimental $\boldsymbol{F}_{\text {total }}$. In the literature [7], the force at the needle tip and the friction force on the needle side were separately measured, and the tip forces were almost flat after the needle tip penetrated the surface, while the friction forces proportionally increased. These tendencies were achieved in our results, because $\boldsymbol{F}_{\text {total }}$ without friction could be regarded as the tip force, which was roughly flat along the time, and $\boldsymbol{F}_{\text {total }}$ with friction linearly increased. Considering that $\boldsymbol{F}_{\mathrm{t}}$ was proportional to both $\boldsymbol{F}_{n}$ and the friction coefficient $\mu$, but $\mu$ should be about 1.0 to match the simulation results with the experimental ones, it was supposed that the tightening force $\left(=\boldsymbol{F}_{n}\right)$ of the solid around the needle side was underestimated. We will investigate this reason in the near future.

The accuracy of the EH was theoretically the same order as that of Lagrangian FEM as far as inside of the solid except the border because of the common Lagrangian step. However, the EH has additional advantages. Considering that medical images consist of voxel data, the data transfer from image to $\mathrm{EH}$ analysis will be easier. In addition, the EH can achieve the strong coupling analysis between solid and fluid by coupling the Lagrangian step of EH to that of fluid dynamics. This might be useful to analyze the deformation of wet tissues. We will improve the EH to be more accurate for biological applications. The next version of the EH has been developed to handle multiple objects. We will try to simultaneously simulate the needle deflection and tissue deformation at the next step.

\section{Conclusions}

In this paper, the resistance forces accompanied with needle insertion were simulated by using a novel Eulerian hydrocode. The simulation results were compared with those of the experiments with silicon rubbers. It was shown that the Eulerian hydrocode could accurately predict the resistance forces as far as the conical portion of the needle was outside the rubber. After the conical portion was inside the rubber, the resistance forces predicted by the simulations were smaller than the experimental ones. It was considered that this difference was caused by the underestimation of the tightening force along the needle side. However, the tendency of the tip forces along the time in the simulations presented a roughly constant as those in the experiments, and the friction force along the needle side presented linear increase along the time. 


\section{References}

1. Okamura, A., Simone, C., O'leary, M.: Force Modeling for Needle Insertion Into Soft Tissue. IEEE Trans. on Biomedical Engineering 51(10), 1707-1716 (2004)

2. DiMaio, S., Salcudean, S.: Needle Steering and Model-Based Trajectory Planning. In: Ellis, R.E., Peters, T.M. (eds.) MICCAI 2003. LNCS, vol. 2879, pp. 33-40. Springer, Heidelberg (2003)

3. Benson, D.J.: Computational Methods in Lagrangean and Eulerian hydrocodes. Comput. Methods Appl. Mech. Engrg. 99, 235-394 (1992)

4. Benson, D.J.: Contact in a Multi-material Eulerian Finite Element Formulation. Comput. Methods Appl. Mech. Engrg. 193, 4277-4298 (2004)

5. Kurganov, A., Levy, D.: A Third-order Semidiscrete Central Scheme for Conservation Laws and Convection-Diffusion Equations. SIAM J. Sci. Comput. 22(4), 1461-1488 (2000)

6. Mofidi, M., Prakash, B., et al.: Rubber Friction on (Apparently) Smooth Lubricated Surfaces. J. Phys. Condens. Matter 20, 085223(8pp) (2008)

7. Washio, K.H., et al.: Measurement of Tip and Friction Force Acting on a Needle During Penetration. In: Dohi, T., Kikinis, R. (eds.) MICCAI 2002. LNCS, vol. 2489, pp. 216-223. Springer, Heidelberg (2002) 